\title{
Persistent Depressive Symptoms after Acute Coronary Syndrome Are Associated with Compromised White Matter Integrity in the Anterior Cingulate: A Pilot Study
}

\author{
Michael A. Rapp ${ }^{a, e}$ Nina Rieckmann ${ }^{a, f}$ David A. Lessman ${ }^{a} \quad$ Cheuk Y. Tang ${ }^{a}$ b \\ Robert Paulino ${ }^{a}$ Matthew M. Burg ${ }^{d}$ Karina W. Davidson ${ }^{c, d}$ \\ Departments of a Psychiatry, ${ }^{b}$ Radiology and ${ }^{\mathrm{c} M o u n t}$ Sinai Heart, Mount Sinai School of Medicine, \\ and ${ }^{\mathrm{d}}$ Department of Medicine and Psychiatry, Columbia University College of Physicians and Surgeons, \\ New York, N.Y., USA; ${ }^{~}$ Department of Psychiatry and ${ }^{\mathrm{f} B e r l i n}$ School of Public Health, Charité University \\ Medical Center, Berlin, Germany
}

\section{Key Words}

Depression · Acute coronary syndrome $\cdot$ White matter lesions • Diffusion tensor imaging

\begin{abstract}
Background: Persistent depressive symptoms after acute coronary syndrome (ACS) are common and increase the risk of recurrent cardiac events and mortality. However, the neurobiological correlates of post-ACS depressive symptoms have not yet been studied. Methods: Three months after ACS, 22 patients were scanned for the presence of cerebral deep white matter changes and microstructural abnormalities in the anterior cingulate cortex (ACC) and dorsolateral prefrontal cortex. We used the Coffey Rating Scale of deep white matter changes and measures of fractional anisotropy derived from diffusion tensor imaging. Patients also completed the Beck Depression Inventory, and the number of cardiovascular comorbidities as well as modifiable cardiovascular risk factors were assessed. Results: Controlling for cardiovascular comorbidity, depressive symptom severity at 3 months was negatively related to fractional anisotropy in
\end{abstract}

\section{KARGER}

Fax +4161306 1234 E-Mail karger@karger.ch www.karger.com
두 2010 S. Karger AG, Basel

www.karger.com/pps the ACC $(r=-0.72, p<0.001)$, but this association disappeared when controlling for cardiovascular risk factors $(p=$ 0.21 ). In comparison to patients who were non-depressed at 3 months after hospitalization ( $n=14)$, patients with persistent depressive symptoms $(n=8)$ exhibited more advanced deep white matter changes overall $(p<0.02)$, but not when controlling for cardiovascular comorbidity. Persistently depressed patients also had lower fractional anisotropy in the ACC $(p<0.05)$, but this effect disappeared when controlling for modifiable cardiovascular risk factors. Conclusions: This study provides the first evidence that persistent depressive symptoms after ACS are associated with vascular brain changes. Longitudinal studies are needed to determine whether depressive symptoms precede these changes or vice versa.

Copyright $\odot 2010$ S. Karger AG, Basel

M.A.R. and N.R. contributed equally to the manuscript. 


\section{Introduction}

Structural MRI studies in elderly patients with vascular depression $[1,2]$ have reported increased deep white matter lesions $[3,4]$; however, white matter changes have also been reported in elderly patients with depression onset at an earlier age [5], and may extend to younger age groups [6]. Specifically, white matter changes in the anterior cingulate circuit [7], and the dorsolateral prefrontal cortex (DLPFC) have been identified in vascular depression $[3,4,6-8]$. These brain areas have been implicated in emotional regulation in humans [9], suggesting a direct link between the structural changes caused by vascular lesions and subsequent negative mood states.

After acute coronary syndrome (ACS), depressive symptoms are common $[10,11]$. In several studies and 2 meta-analyses, depressive symptoms emerged as reliable predictors of subsequent all-cause mortality as well as rehospitalization for cardiac events, even at low levels of depression [12-14]. This relationship follows a dose-response fashion - the more severe the depressive symptoms, the higher the risk [15] - and there is some evidence that patients with depressive symptoms that persist across several months appear to be at highest risk for poor outcomes $[12,16,17]$.

Several biological correlates of depression and vascular disease have been proposed to explain the risk conferred by persistently elevated depressive symptoms [for review, see 18], including elevated inflammatory markers [19] and dysregulation of the hypothalamic-pituitary-adrenal axis with subsequent changes in autonomic cardiac function [20]. However, the neurobiological correlates of post-ACS depressive symptoms have not yet been studied empirically. Studies showing a high comorbidity between cardiovascular disease and depression - in which about $30 \%$ of patients experience elevated depressive symptoms after an ACS and 20\% have major depressive disorder [21] - suggest that at least in some patients, depressive symptoms may be of vascular origin. Using MRI, vascular lesions have traditionally been assessed using standardized rating scales [22] that quantify vascular changes at the macrostructural level. An alternative method is diffusion tensor imaging, which employs MRI techniques to measure alterations in brain tissue microstructure [23].

We explored the relationship between depressive symptoms after an ACS (assessed at hospitalization and after 3 months) and macro- and microstructural abnormalities in the brain (assessed 3 months after the ACS). Specifically, we assessed the relationship between the se- verity of depressive symptoms and measures of fractional anisotropy in the anterior cingulate cortex (ACC) and DLPFC, and the extent of deep white matter hyperintensities. We further compared post-ACS patients who had persistently elevated depressive symptoms to those who had no or only transient depressive symptoms on the same measures.

\section{Methods}

\section{Participants}

Participants were a subsample of the Coronary Psychosocial Evaluation Studies (COPES) that included a consecutive sample of ACS patients who were admitted to the coronary care units of 3 university hospitals between May 1, 2003 and June 13, 2005. Diagnosis of ACS was determined by research criteria [for details, see 24]. Patients were included in the study if they met pre-specified depressive symptom levels according to the Beck Depression Inventory (BDI [13]), i.e. scores $\geq 10$ or $<5$. A BDI score of 10 or higher indicates elevated depressive symptoms, and has been associated with increased mortality and recurrent cardiac event risk after ACS [11].

Patients were recruited after Institutional Review Board approval and informed consent. Recruitment for the MRI substudy took place at the Mount Sinai site only; of the 189 patients enrolled at the site, only $36(19 \%)$ agreed to take part in the MRI study at 3 months. Of these, 14 participants were excluded because of metallic implants other than cardiac stents $(n=2)$, claustrophobia $(n=2)$, active alcohol or substance abuse $(n=2)$, cognitive impairment as indicated by a Mini-Mental State Examination (MMSE [25]) score $<24(\mathrm{n}=1)$, scanner downtime $>4$ weeks $(\mathrm{n}=$ $3)$, and poor data quality $(n=3)$. The remaining sample $(n=22)$ did not differ from the parent sample in terms of age, sex, ethnicity, BMI, smoking, the number of cardiovascular diagnoses, and depression history (all $\mathrm{p}>0.21$ ).

\section{Clinical Variables}

At baseline, within 1 week of hospitalization for ACS, patients completed a demographic form assessing age, sex, and ethnicity. Height and weight were measured, and their smoking status assessed (never smoked vs. ever smoked vs. non-smoker; in pack years). They were administered the MMSE [25] and the BDI [26]. The BDI was re-administered 3 months after enrollment. Patients who had a score of $\geq 10$ on the BDI at both baseline (in hospital) and after 3 months were considered 'persistently depressed'.

All participants also underwent a semi-structured diagnostic interview (Depression Interview and Structured Hamilton [27]) to determine the presence of major and minor depressive disorder according to the DSM-IV criteria, including lifetime history of depressive illness [28]. Previous cardiovascular and metabolic diagnoses (history of peripheral vascular disease, hypercholesterolemia, diabetes, recurrent angina, history of hypertension, congestive heart failure) were collected from medical charts and summed. Severity of the index ACS was coded according to clinical criteria [29] as unstable angina, ST-segment elevation myocardial infarction, and non-ST-segment elevation myocardial infarction. Treatment of the index ACS episode was assessed in 5 catego- 
ries (no treatment; cardiac catheterization; thrombolysis; coronary artery bypass graft; other). The course of the index ACS during the first 3 months was coded dichotomously as a function of the number of clinical interventions (emergency room visits; hospitalizations; cardiac catheterization; thrombolysis; coronary artery bypass graft; other) needed during the follow-up period ( $0=$ no intervention needed; 1 = one or more interventions needed).

\section{MRI Procedures}

Scanning was performed using a 1.5-T Siemens Vision Scanner at the Mount Sinai School of Medicine. Patients received an MPRAGE (3D-magnetization-prepared rapidly acquired gradient echo) scan [repetition time $(\mathrm{TR})=2,500 \mathrm{~ms}$, echo time $(\mathrm{TE})=4.38 \mathrm{~ms}$, matrix $=256 \times 256$, field of view $(\mathrm{FOV})=210$ $\mathrm{mm}$, number of excitations $(\mathrm{NEX})=1$, slice thickness $=0.8 \mathrm{~mm}$, 208 slices, no gap, inversion time $(\mathrm{TI})=1,100 \mathrm{~ms}$, a fast dual-spin echo scan $(\mathrm{TR}=6,250 \mathrm{~ms}, \mathrm{TE}=14 / 96 \mathrm{~ms}$, matrix $=256 \times 256$, FOV $=210 \mathrm{~mm}$, slice thickness $=3 \mathrm{~mm}, 45$ slices, no gap), a FLAIR (fluid-attenuated inversion recovery) $\operatorname{scan}(\mathrm{TR}=8,340 \mathrm{~ms}$, TE $=$ $2,500 \mathrm{~ms}$, matrix $=320 \times 320, \mathrm{FOV}=230 \mathrm{~mm}, \mathrm{NEX}=1$, slice thickness $=3 \mathrm{~mm}, 40$ slices, no gap), and a diffusion tensor scan $(\mathrm{TR}=5,100 \mathrm{~ms}, \mathrm{TE}=80 \mathrm{~ms}$, matrix $=128 \times 128, \mathrm{FOV}=220 \mathrm{~mm}$, NEX $=7$, slice thickness $=3 \mathrm{~mm}, 40$ slices, no gap, $\mathrm{b}=1,250$ $\mathrm{s} / \mathrm{mm}^{2}$ ). Twelve diffusion sensitization directions were used.

\section{Coffey Scale}

The Coffey classification system [22] was used to describe the extent of deep white matter hyperintensities on anatomical scans. This grading system rates deep white matter lesions on a 4-point scale (absent, punctuate, confluent, or large). Two raters (M.A.R., D.A.L.), who were blinded to group membership, rated the scans. Inter-rater reliability was satisfactory $(r=0.83)$.

\section{Image Processing}

In-house software (CYT) was used to compute the anisotropy and vector maps. MEDx v3.4.3 software (Medical Numerics Inc., Sterling, Va., USA) was used to inspect and define regions of interest (ROIs) in the ACC and DLPFC on the fractional anisotropy images. Two adjacent axial planes were selected to contain the same voxel locations. ROI dimensions for the fractional anisotropy images were set to $3 \times 3$. Each 3 ROIs were placed in ACC and DLPFC. Fractional anisotropy values of the white matter underlying each ROI were averaged for each area and extracted. Fractional anisotropy data were missing for 3 patients (2 did not complete scanning, 1 due to accidental data loss).

\section{Statistical Analyses}

The $\chi^{2}$ test for categorical variables and t test for continuous variables were used to assess depression group differences in descriptive variables and covariates. The associations between depression severity, depression groups, and Coffey scores were examined using non-parametric statistics (Kendall's tau $u_{b}$ and $\chi^{2}$ test). For non-parametric analyses of covariance, we used a logistic regression approach to control for covariates at each dichotomous category step (e.g. for the Coffey scale, absent vs. punctuate, punctuate vs. confluent, and confluent vs. large) and then compared the residuals as a function of depression group using $\chi^{2}$ tests [for a similar approach, see 30]. Fractional anisotropy measures were treated as continuous variables; bivariate Pearson correlations were used to assess the relation between fractional anisot- ropy measures and depressive symptom severity, and hierarchical linear regression was used to control for potential covariates. Univariate ANOVA was used to assess depression group differences in fractional anisotropy measures; analysis of covariance was used to control for covariates. Two-tailed tests of significance were employed with $\alpha$ set at $<0.05$. All analyses were performed using statistical software (SPSS Inc., Chicago, Ill., USA).

\section{Results}

\section{Demographic and Clinical Characteristics}

The overall study group $(\mathrm{n}=22)$ comprised 9 women (40.9\%) and 13 men, with a mean age of 56.09 years $(\mathrm{SD}=9.53)$. At baseline, 16 patients had significant depressive symptoms, and 6 were non-depressed. Eight patients remained persistently depressed after 3 months (BDI score $\geq 10$ ), the other 14 remained non-depressed or remitted to a BDI score $<10$ by 3 months. No patient developed new significant depressive symptoms during that time.

Table 1 displays the demographic characteristics of persistently depressed patients in comparison to patients without depression or those with transient depressive symptoms only.

\section{White Matter Lesions (Coffey Scale)}

Depressive symptoms at 3 months were significantly correlated with white matter lesions $(\tau=0.41, \mathrm{p}<0.02)$. A similar albeit weaker and non-significant correlation was observed for depressive symptoms at baseline $(\tau=$ $0.20, p=0.24$ ).

Patients with persistent depressive symptoms exhibited more advanced deep white matter changes, as assessed by the Coffey Scale, compared to post-ACS patients with no or transient depressive symptoms $\left(\chi^{2}=5.25\right.$, d.f. $=1, \mathrm{p}<$ 0.02 ; fig. 1). This difference remained significant when adjusting for age, sex, ethnicity, BMI, and smoking status $\left(\chi^{2}=4.47\right.$, d.f. $\left.=1, p<0.03\right)$. When controlling for the number of cardiovascular diagnoses, including diabetes, however, this effect diminished ( $\mathrm{p}=0.31$ ). Neither the type of treatment $(\mathrm{p}=0.34)$ nor the severity $(\mathrm{p}=0.18)$ nor the course $(\mathrm{p}=0.48)$ of the ACS index episode had an effect on deep white matter changes. In addition, the presence versus absence of a lifetime history of depression did not have an effect on deep white matter changes $(\mathrm{p}=0.21)$.

\section{Fractional Anisotropy in the ACC and the \\ Dorsolateral Prefrontal Circuit}

Fractional anisotropy in the ACC was significantly related to depression severity at 3 months $(r=-0.72, p<$ 0.001 ; fig. 2). This association remained significant when 


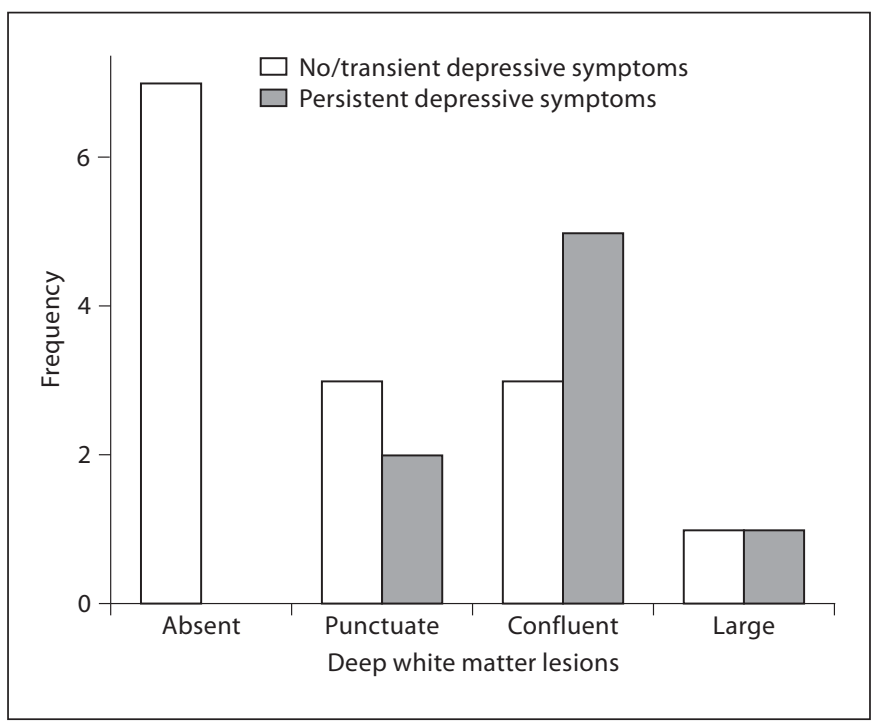

Fig. 1. Deep white matter lesions as a function of depressive symptom stability 3 months after ACS.

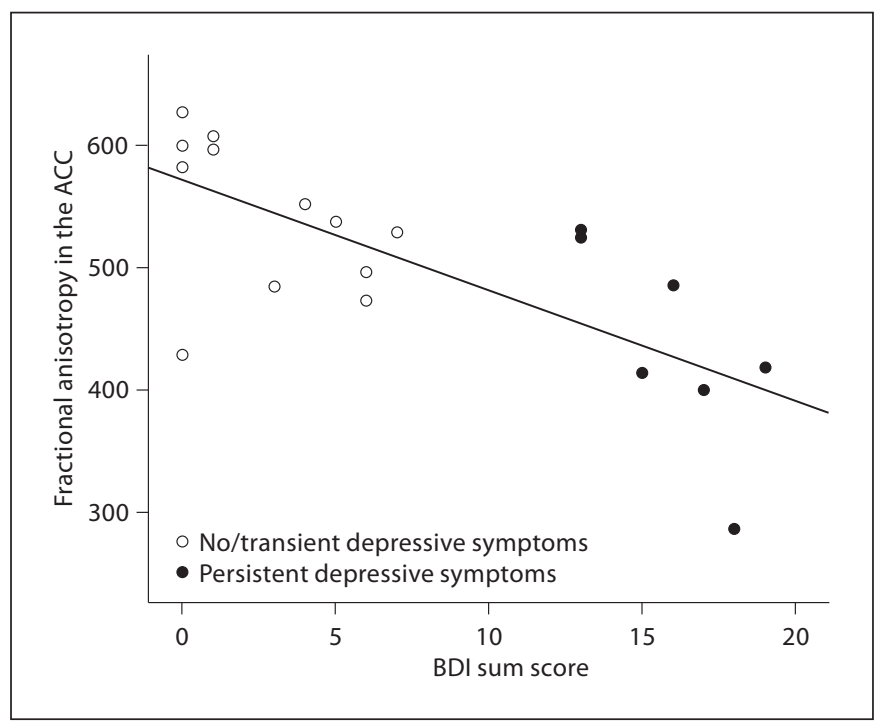

Fig. 2. Relationship between depression severity and fractional anisotropy in the ACC at 3 months after ACS. Three patients are missing fractional anisotropy data $(\mathrm{n}=19$, see 'Methods').

Table 1. Demographic and clinical characteristics of patients with no/transient depressive symptoms and patients with persistent depressive symptoms

No/transient depressive symptoms $(\mathrm{n}=14)$

Age, years

Females

Hispanic

Smoking, pack years

MMSE score

Previous cardiovascular diagnoses (including diabetes) Index ACS

Unstable angina

Non-ST-segment elevation myocardial infarction

ST-segment elevation myocardial infarction

Treatment of index ACS

Catheterization

Thrombolysis

Coronary artery bypass surgery

Other

Interventions needed in first 3 months after ACS

Body mass index

BDI score at 3 months

Depressive disorder status at 3 months

Minor depression

Major depressive episode

History of depression

$$
\begin{aligned}
56.14 & \pm 10.60 \\
4 & (28.6) \\
3 & (21.4) \\
21.94 & \pm 18.49 \\
29.79 & \pm 0.43 \\
3.15 & \pm 4.86 \\
& 6(42.9) \\
2 & (14.3) \\
6 & (42.9) \\
9 & (64.3) \\
4 & (28.6) \\
1 & (7.1) \\
0 & (0.0) \\
11 & (78.6) \\
26.34 & \pm 2.4 \\
2.86 & \pm 2.89
\end{aligned}
$$

0

0

$7(50.0)$
Persistent depressive symptoms $(\mathrm{n}=8)$

$\begin{array}{cc}56.0 \pm 8.0 & 0.97 \\ 5(62.5) & 0.19 \\ 5(62.5) & 0.08 \\ 24.60 \pm 36.89 & 0.85 \\ 29.13 \pm 1.25 & 0.08 \\ 2.63 \pm 1.23 & 0.77 \\ & \\ 5(62.5) & \\ 2(25.0) & 0.34 \\ 1(12.5) & \\ & \\ 4(50.0) & \\ 3(37.5) & \\ 0(0.0) & 0.62 \\ 1(12.5) & 0.60 \\ 7(87.5) & 0.001 \\ 32.86 \pm 5.34 & <0.001 \\ 16.88 \pm 3.60 & \\ & <0.001 \\ 5(62.5) & 0.67 \\ 2(25.0) & \end{array}$

Data represent means $\pm \mathrm{SD}$ or $\mathrm{n}(\%)$. $\mathrm{p}$ 0.97 0.19 0.85 0.08 0.77 0.34 0.60 0.001 $<0.001$ 
controlling for sex, ethnicity, smoking status, body mass index, and number of cardiovascular diagnoses $(\beta=$ $-0.66, p<0.05)$. Fractional anisotropy in the ACC was not, however, significantly related to depressive symptom severity at baseline $(r=-0.23, p=0.34)$. Neither the type of treatment, nor the severity, nor the course of the ACS index episode had an effect on fractional anisotropy in the ACC (all $p>0.32$ ) or DLPFC (all $p>0.53$ ). In addition, the presence versus absence of a lifetime history of depression did not have an effect on fractional anisotropy ( $\mathrm{p}=$ 0.27).

In comparison to patients with no or only transient depressive symptoms, patients with persistent depressive symptoms had significantly lower fractional anisotropy in the $\operatorname{ACC}\left(F_{1,17}=9.74, p<0.01, \eta^{2}=0.36\right)$. This difference remained significant when controlling for age, sex, ethnicity, and the number of vascular diagnoses, including diabetes, and the severity, course, and treatment of the index ACS $\left(F_{6,13}=5.29, \mathrm{p}<0.05, \eta^{2}=0.31\right)$, but disappeared when controlling for modifiable risk factors of $\mathrm{BMI}$ and smoking in pack years $(\mathrm{p}=0.28)$. There was no association between depressive symptom severity or depression group and lower fractional anisotropy in the DLPFC ( $\mathrm{p}=0.46$ and 0.97 , respectively).

\section{Discussion}

We found distinct differences in macrostructural deep white matter changes in post-ACS patients as assessed by semi-quantitative ratings (Coffey Scale [22]) as a function of depressive symptom severity and persistence after 3 months. This difference remained significant when controlling for modifiable risk factors, but disappeared when controlling for the number of cardiovascular diseases. Furthermore, we found a significant relationship between fractional anisotropy, reflecting microstructural changes in the ACC, and depressive symptom severity and persistence. This association remained significant when controlling for cardiovascular diseases; however, when controlling for modifiable risk factors, this association was not significant anymore.

Our findings show that depression after ACS is associated with vascular brain lesions in an area critical to the pathophysiology of depression [7-9], suggesting that in some patients, post-ACS depression is related to vascular lesions consistent with models of vascular depression $[1,2]$ and earlier findings of depression persistence in the presence of vascular brain changes, both on the microstructural $[7,8]$ and the macrostructural $[2-4]$ levels. We propose that white matter changes on the micro- and macrostructural levels may both contribute to depression vulnerability [11] in post-ACS patients, may perpetuate depression beyond adjustment processes after ACS $[12,13]$, and may be a critical biomarker for the depression-mortality association reported after ACS [11-16]. Our findings suggest that the severity of cardiovascular disease mediates the association between depression and macrostructural changes, whereas modifiable risk factors mediate the association between depression and microstructural changes. However, it has to be noted that directional causality cannot be inferred from our data, and there is indeed evidence that depression may precede cerebrovascular lesions and risk factors $[31,32]$.

Several limitations have to be considered. First, we cannot rule out the possibility that other biological mechanisms, such as inflammatory response [19] and dysregulation of the hypothalamic-pituitary-adrenal axis with subsequent changes in autonomic cardiac function [20], may be related to vascular changes in post-ACS depression, since we lack these data in our study. Second, the extent of cerebrovascular lesions could be related to the severity of cardiovascular comorbidities, but we lack specific data on the severity of these diagnoses in our sample. Treatment of the index ACS, however, including the use of stenting, did not explain the observed differences in cerebrovascular lesions in our study. Third, this study is limited by its small sample size and limited number of patients with major depressive disorder. This did not allow us to examine the contribution of specific depressive disorder subtypes that have recently been shown to predict post-ACS mortality and event recurrence, such as the distinction between incident and recurrent major depressive episode $[33,34]$. In that realm, the study provides only preliminary data and is of pilot nature.

Given the finding that cardiovascular diagnoses account for a significant amount of variance in macrostructural (deep white matter) changes, while cardiovascular risk factors account for a significant amount of variance in microstructural changes (fractional anisotropy), one may speculate that modifiable risk factors may have a specific impact on microstructural changes associated with persistent depression. Recently, it has been proposed that depressive symptomatology lies along a continuum of subsyndromal and prodromal stages of depressive disorders [35]. In light of our finding of an association between cerebrovascular lesions and depressive symptomatology, even below the threshold for major depressive disorder [26], such a continuum may well be related to 
continuous alterations on a neurobiological level. Furthermore, it has been suggested that residual symptoms of depression may be especially amenable to non-pharmacological interventions beyond pharmacological treatment effects [36], and this insight may be especially valuable in the study of post-ACS depression, since additional factors - such as medication adherence [37], lifestyle adaptations to mitigate behavioral risk factors, and adherence to other preventive measures - may strongly depend upon individually tailored non-pharmacological interventions in this population [38].

Despite its preliminary nature, this study establishes post-ACS depressive symptoms as 'vascular' depression $[1,2]$ consistent with deep white matter hyperintensities and microstructural white matter abnormalities in the ACC. The identification of the neurobiological correlates of post-ACS depression can guide further investigation aimed at enhancing therapeutic interventions and disentangling causal mechanisms in the relationship between depression and cardiovascular disease.

\section{Acknowledgments and Conflict of Interest}

This work was supported by grants HC-25197, HL-76857, and HL-84034 from the National Heart, Lung, and Blood Institute, Bethesda, Md., USA, and by grant No. UL1 RR024156 from the National Center for Research Resources (NCRR; www.ncrr.nih. gov), a component of the National Institutes of Health (NIH) and NIH Roadmap for Medical Research. This report is solely the responsibility of the authors, and does not necessarily represent the official view of NCRR or NIH. Information on Re-engineering the Clinical Research Enterprise can be obtained from http://nihroadmap.nih.gov/clinicalresearch/overview-translational.asp.

M.A.R. has received speaker honoraria and compensation from Janssen Cilag AG, Glaxo Smith Kline, Inc., and Servier, Inc.

\section{References}

- 1 Alexopoulos GS, Meyers BS, Young RC, Campbell S, Silbersweig D, Charlson M: 'Vascular depression' hypothesis. Arch Gen Psychiatry 1997;54:915-922.

-2 Krishnan KR, Hays JC, Blazer DG: MRI-defined vascular depression. Am J Psychiatry 1997;154:497-501.

>3 de Groot JC, de Leeuw FE, Oudkerk M, Hofman A, Jolles J, Breteler MM: Cerebral white matter lesions and depressive symptoms in elderly adults. Arch Gen Psychiatry 2000;57: 1071-1076.

4 Taylor WD, MacFall JR, Payne ME, McQuoid DR, Steffens DC, Provenzale JM, Krishnan RR: Greater MRI lesion volumes in elderly depressed subjects than in control subjects. Psychiatry Res 2005;139:1-7.

-5 Kumar A, Bilker W, Jin Z, Udupa J, Gottlieb G: Age of onset of depression and quantitative neuroanatomic measures: absence of specific correlates. Psychiatry Res 1999;91: 101-110.

-6 Hickie I, Scott E, Mitchell P, Wilhelm K, Austin MP, Bennett B: Subcortical hyperintensities on magnetic resonance imaging: clinical correlates and prognostic significance in patients with severe depression. Biol Psychiatry 1995;37:151-160.

7 Bae JN, MacFall JR, Krishnan KR, Payne ME, Steffens DC, Taylor WD: Dorsolateral prefrontal cortex and anterior cingulate cortex white matter alterations in late-life depression. Biol Psychiatry 2006;60:13561363.
-8 Murphy CF, Gunning-Dixon FM, Hoptman MJ, Lim KO, Ardekani B, Shields JK, Hrabe J, Kanellopoulos D, Shanmugham BR, Alexopoulos GS: White-matter integrity predicts Stroop performance in patients with geriatric depression. Biol Psychiatry 2007;61:1007-1010.

-9 Mayberg HS: Limbic-cortical dysregulation: a proposed model of depression. J Neuropsychiatry Clin Neurosci 1997;9:471-481.

10 Davidson KW, Kupfer DJ, Bigger JT, Califf RM, Carney RM, Coyne JC, Czajkowski SM, Frank E, Frasure-Smith N, Freedland KE, Froelicher ES, Glassman AH, Katon WJ, Kaufmann PG, Kessler RC, Kraemer HC, Krishnan KR, Lespérance F, Rieckmann N, Sheps DS, Suls JM, National Heart, Lung, and Blood Institute Working Group: Assessment and treatment of depression in patients with cardiovascular disease: National Heart, Lung, and Blood Institute Working Group Report. Ann Behav Med 2006;32:121-126.

11 Rieckmann N, Burg MM, Gerin W, Chaplin WF, Clemow L, Davidson KW: Depression vulnerabilities in patients with different levels of depressive symptoms after acute coronary syndromes. Psychother Psychosom 2006;75:353-361.

12 Lespérance F, Frasure-Smith N, Talajic M Bourassa MG: Five-year risk of cardiac mortality in relation to initial severity and oneyear changes in depression symptoms after myocardial infarction. Circulation 2002, 105:1049-1053.
-13 van Melle JP, de Jonge P, Spijkerman TA, Tijssen JG, Ormel J, van Veldhuisen DJ, van den Brink RH, van den Berg MP: Prognostic association of depression following myocardial infarction with mortality and cardiovascular events: a meta-analysis. Psychosom Med 2004;66:814-822.

14 Nicholson A, Kuper H, Hemingway H: Depression as an aetiologic and prognostic factor in coronary heart disease: a metaanalysis of 6362 events among 146538 participants in 54 observational studies. Eur Heart J 2006;27:2763-2774.

15 Thombs BD, Ziegelstein RC, Stewart DE, Abbey SE, Parakh K, Grace SL: Usefulness of persistent symptoms of depression to predict physical health status 12 months after an acute coronary syndrome. Am J Cardiol 2008;101:15-19.

16 Rafanelli C, Roncuzzi R, Milaneschi Y, Tomba E, Colistro MC, Pancaldi LG, Di Pasquale G: Stressful life events, depression and demoralization as risk factors for acute coronary heart disease. Psychother Psychosom 2005;74:179-184.

-17 Parashar S, Rumsfeld JS, Spertus JA, Reid KJ, Wenger NK, Krumholz HM, Amin A, Weintraub WS, Lichtman J, Dawood N, Vaccarino $\mathrm{V}$ : Time course of depression and outcome of myocardial infarction. Arch Intern Med 2006; 166:2035-2043.

18 Carney RM, Freedland KE: Treatment-resistant depression and mortality after acute coronary syndrome. Am J Psychiatry 2009; $166: 410-417$ 
-19 Shimbo D, Rieckmann N, Paulino R, Davidson KW: Relation between $\mathrm{C}$ reactive protein and depression remission status in patients presenting with acute coronary syndrome. Heart 2006;92:1316-1318.

-20 de Guevara MS, Schauffele SI, Nicola-Siri LC, Fahrer RD, Ortiz-Fragola E, MartinezMartinez JA, Cardinali DP, Guinjoan SM: Worsening of depressive symptoms 6 months after an acute coronary event in older adults is associated with impairment of cardiac autonomic function. J Affect Disord 2004;80:257-262.

-21 Thombs BD, Bass EB, Ford DE, Stewart KJ, Tsilidis KK, Patel U, Fauerbach JA, Bush DE, Ziegelstein RC: Prevalence of depression in survivors of acute myocardial infarction. J Gen Intern Med 2006;21:30-38.

$\checkmark 22$ Coffey CE, Figiel GS, Djang WT, Saunders WB, Weiner RD: White matter hyperintensity on magnetic resonance imaging: clinical and neuroanatomic correlates in the depressed elderly. J Neuropsychiatry Clin Neurosci 1989;1:135-144.

-23 Buchsbaum MS, Tang CY, Peled S, Gudbjartsson H, Lu D, Hazlett EA, Downhill J, Haznedar M, Fallon JH, Atlas SW: MRI white matter diffusion anisotropy and PET metabolic rate in schizophrenia. Neuroreport 1998;9:425-430.

-24 Frasure-Smith N, Lespérance F, Talajic M: The impact of negative emotions on prognosis following myocardial infarction: is it more than depression? Health Psychol 1995; $14: 388-398$.
25 Folstein MF, Folstein SE, McHugh PR: 'MiniMental State': a practical method for grading the cognitive state of patients for the clinician. J Psychiatr Res 1975;12:189-198.

-26 Beck AT, Guth D, Steer RA, Ball R: Screening for major depression disorders in medical inpatients with the Beck Depression Inventory for Primary Care. Behav Res Ther 1997;35: 785-791.

27 Freedland KE, Skala JA, Carney RM, Raczynski JM, Taylor CB, Mendes de Leon CF, Ironson G, Youngblood ME, Krishnan KR, Veith RC: The Depression Interview and Structured Hamilton (DISH): rationale, development, characteristics, and clinical validity. Psychosom Med 2002;64:897-905.

28 American Psychiatric Association: Diagnostic and Statistical Manual of Mental Disorders, DSM-IV. Washington, American Psychiatric Press, 1994.

29 Manoharan G, Ntalianis A, Muller O, Hamilos M, Sarno G, Melikian N, Vanderheyden M, Heyndrickx GR, Wyffels E, Wijns W, De Bruyne B: Severity of coronary arterial stenoses responsible for acute coronary syndromes. Am J Cardiol 2009;103:1183-1188.

30 Tsangari H, Akritas MG: Nonparametric ANCOVA with two and three covariates. J Multivar Anal 2004;88:298-319.

- 31 Krishnan KR: Depression as a contributing factor in cerebrovascular disease. Am Heart J 2000;140:70-76.

-32 Krishnan M, Mast BT, Ficker LJ, Lawhorne L, Lichtenberg PA: The effects of preexisting depression on cerebrovascular health outcomes in geriatric continuing care. J Gerontol A Biol Sci Med Sci 2005;60:915-919.
3 de Jonge $\mathrm{P}$, van den Brink RH, Spijkerman TA, Ormel J: Only incident depressive episodes after myocardial infarction are associated with new cardiovascular events. J Am Coll Cardiol 2006;48:2204-2208.

34 Parker GB, Hilton TM, Walsh WF, Owen CA, Heruc GA, Olley A, Brotchie H, HadziPavlovic D: Timing is everything: the onset of depression and acute coronary syndrome outcome. Biol Psychiatry 2008;64:660-666.

35 Hetrick SE, Parker AG, Hickie IB, Purcell R, Yung AR, McGorry PD: Early identification and intervention in depressive disorders: towards a clinical staging model. Psychother Psychosom 2008;77:263-270.

36 Fava GA, Tomba E, Grandi S: The road to recovery from depression - don't drive today with yesterday's map. Psychother Psychosom 2007;76:260-265.

37 Rieckmann N, Gerin W, Kronish IM, Burg MM, Chaplin WF, Kong G, Lespérance F, Davidson KW: Course of depressive symptoms and medication adherence after acute coronary syndromes: an electronic medication monitoring study. J Am Coll Cardiol 2006;48:2218-2222.

38 Burg MM, Lespérance F, Rieckmann N, Clemow L, Skotzko C, Davidson KW: Treating persistent depressive symptoms in postACS patients: the project COPES phase-I randomized controlled trial. Contemp Clin Trials 2008;29:231-240. 